\title{
Topographic Mapping as a Basic Principle of Functional Organization for Visual and Prefrontal Functional Connectivity
}

\author{
Jonathan F. O'Rawe and Hoi-Chung Leung
}

https://doi.org/10.1523/ENEURO.0532-19.2019

Integrative Neuroscience Program, Department of Psychology, Stony Brook University, Stony Brook, NY 11794-2500

\begin{abstract}
The organization of region-to-region functional connectivity has major implications for understanding information transfer and transformation between brain regions. We extended connective field mapping methodology to 3-D anatomic space to derive estimates of corticocortical functional organization. Using multiple publicly available human (both male and female) resting-state fMRI data samples for model testing and replication analysis, we have three main findings. First, we found that the functional connectivity between early visual regions maintained a topographic relationship along the anterior-posterior dimension, which corroborates previous research. Higher order visual regions showed a pattern of connectivity that supports convergence and biased sampling, which has implications for their receptive field properties. Second, we demonstrated that topographic organization is a fundamental aspect of functional connectivity across the entire cortex, with higher topographic connectivity between regions within a functional network than across networks. The principle gradient of topographic connectivity across the cortex resembled whole-brain gradients found in previous work. Last but not least, we showed that the organization of higher order regions such as the lateral prefrontal cortex demonstrate functional gradients of topographic connectivity and convergence. These organizational features of the lateral prefrontal cortex predict task-based activation patterns, particularly visual specialization and higher order rules. In sum, these findings suggest that topographic input is a fundamental motif of functional connectivity between cortical regions for information processing and transfer, with maintenance of topography potentially important for preserving the integrity of information from one region to another.
\end{abstract}

Key words: fMRI; functional connectivity; functional organization; resting state; spatial topography

\section{Significance Statement}

Quantifying spatial patterns of region-to-region functional connectivity provides an avenue for testing theories of corticocortical information transformation and organization. This work demonstrates that this quantification is feasible not only in early visual cortex, but even in highly multimodal regions where spatial topography is less clear. Overall, we show that topographic relationships as a common motif functional connectivity across the cortex between regions within the same functional network and that analyzing the lateral prefrontal cortex in terms of topographic connectivity reveals organizational features that voxel-wise connectivity analysis misses.

Received December 16, 2019; accepted December 18, 2019; First published January 27, 2020.

The authors declare no competing financial interests.
Author contributions: J.F.O. and H.-C.L. designed research; J.F.O. performed research; J.F.O. contributed unpublished reagents/analytic tools; J.F.O. analyzed data; J.F.O. and H.-C.L. wrote the paper. 


\section{Introduction}

One primary goal of systems neuroscience is to understand how integration occurs across cortical areas in order to support complex behavior (Cavada and GoldmanRakic, 1991; Hoover and Strick, 1993; Haber et al., 2000). As different brain regions display spatially specific patterns of long range and short range connections to their target regions (Van Essen and Gallant, 1994; Kravitz et al., 2011, 2013), it is believed that the particular properties of brain circuitry must provide a foundation for brain function and information processing, and that connectivity profiles from one region to another allows for the potential understanding of information transfer/transformation. Topographic, convergent, and divergent connectivity have been postulated to support different computational needs of a given neural network (Thivierge and Marcus, 2007). For example, systematic convergent connectivity in the visual hierarchy has been shown to be responsible for object processing, from lines (Hubel and Wiesel, 1962) to complex objects (Tanaka, 1997), while divergent connectivity has been theorized in motor systems for complex feedback mechanisms, such as motor efference copies (Wolpert and Flanagan, 2001).

Neuroimaging studies of brain connectivity, while indirect, have the benefit of their exhaustive sampling across the entire anatomic space, which complement the interpretations from the sparse sampling of rigorous anatomic tracing in animals. This is evident in functional connectivity studies using resting state $\mathrm{fMRI}$ data, from which a low dimensional structure of large scale networks has been consistently observed as a key organizational feature of the cortex (Fox et al., 2005; Biswal et al., 2010; Yeo et al., 2011; Di et al., 2013). Indeed, decomposing the whole brain into its fundamental gradients have revealed a structure for the organization of large scale networks, with the primary gradient trending from unimodal networks to multimodal networks (Margulies et al., 2016). However, despite the overwhelming focus on large scale brain organization, detailing the particular patterns of connectivity from one region to another remains a challenge.

Region-to-region topographic connectivity has been observed between early visual areas, which show retinotopic organization (Dumoulin and Wandell, 2008; Wang et al., 2015). By systematically seeding functional connectivity from known functional gradients, it has been shown that functional connectivity within a visual region, and between visual regions seem to follow along known eccentricity organization, along the anterior-posterior axis (Arcaro et al., 2015). In other words, certain parts of a visual region that represent certain eccentricities are more likely to connect to the specific parts of other visual regions that represent similar eccentricities. While this type of systematic seeding is possible in areas with a known and measurable sensory

Correspondence should be addressed to Jonathan F. O'Rawe at jonathan. orawe@gmail.com or Hoi-Chung Leung at hoi-chung.leung@stonybrook.edu.

https://doi.org/10.1523/ENEURO.0532-19.2019

Copyright @ 2020 O'Rawe and Leung

This is an open-access article distributed under the terms of the Creative Commons Attribution 4.0 International license, which permits unrestricted use, distribution and reproduction in any medium provided that the original work is properly attributed. topography, a more general model, such as connective field mapping (Haak et al., 2013), is useful for studying the connection topography of higher order regions. The previous connective field mapping technique fits 2-D surface based population receptive fields (pRFs) using cortical signal from one region as the input to another region (Haak et al., 2013). This method has been used to model early visual areas, and demonstrate maintenance of retinotopic organization across these visual regions (Haak et al., 2013; Gravel et al., 2014).

It is unclear whether the linear pattern of connectivity observed in early visual cortex is dependent on a shared sensory topographic organization, or whether it is present in any highly connected network. In particular, for multimodal regions such as the prefrontal cortex, while it is known that various subdivisions are closely connected, the exact functional organization remains controversial (Ungerleider et al., 1998; Goldman-Rakic, 1999; Petrides, 2005). In theoretical models, spatial topography in connectivity is postulated to serve the function of ensuring the fidelity of information transfer across a network (Thivierge and Marcus, 2007). Topographic organization of connectivity also has the potential to generate complex abstract representations by virtue of topographic overlap (Tinsley, 2009). This suggests that abstract computations can be performed by biases in spatial connectivity and filtering between regions of a large scale network, in the same way that has been suggested in the visual system (Van Essen and Gallant, 1994; Tinsley, 2009).

In this study, we have modified and extended the connective field model, allowing for the estimation of topography of functional connectivity between regions using resting-state $\mathrm{fMRI}$ data in order to infer potential fidelity of information transfer between regions within and across unimodal and multimodal networks. This method fits a 3-D isotropic Gaussian to the connectivity pattern in the mapping region from individual voxels in the seed region (Fig. $1 A)$. This fitting procedure produced four parameters, three location parameters describing the preferred locus of connectivity and a spread parameter, and the fitted Gaussian acts like an encoding model for time courses of BOLD activity (Fig. 1B). We further estimated the degree of topographic mapping from one region to another by performing Procrustes analysis. The degree to which the rank ordered parameters can be fit to the rank ordered coordinates using a linear transformation provides the estimation of the topographic mapping between regions. We first examined the degree of topography connectivity in the visual system, as the literature is relatively established, and then examined whether it is simply a specific case of a general motif of brain functional connectivity across various networks, and finally used this approach to examine prefrontal cortex functional organization.

\section{Materials and Methods}

\section{Subjects}

We used three publicly available datasets: one sample of healthy controls with well validated resting state fMRI data, which is the Cambridge Buckner subset of the 1000 


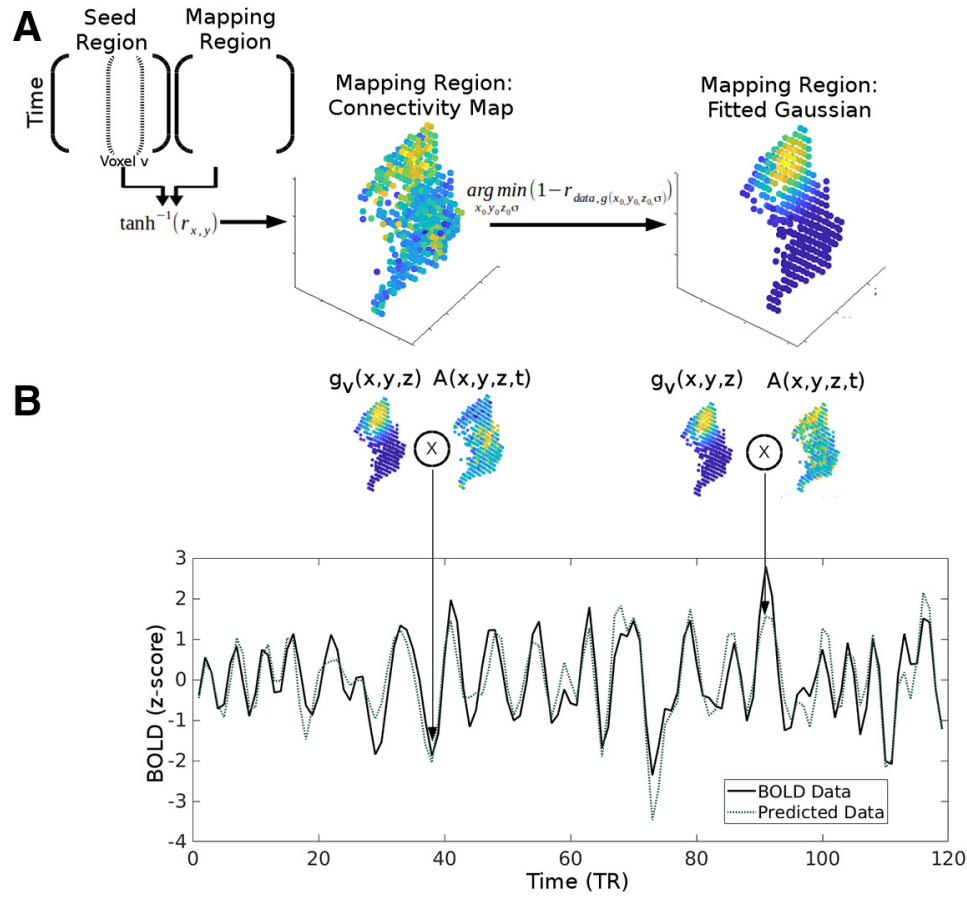

Figure 1. 3-D connective field modeling. $\boldsymbol{A}$, Gaussian fitting procedure. Each seed voxel's time course (dotted bracket inside the matrix on the left represents the time course of one voxel of the seed region) is correlated to every voxel's time course in the mapping region and then Fisher's z transformed. For each seed voxel, the corresponding Fisher's Z matrix is then fitted to the 3-D Gaussian model, by minimizing the correlation distance between the Fisher's Z matrix and the Gaussian distribution. The fitted Gaussian parameters, location, and standard deviation (SD) parameters, for each seed voxel are used in subsequent quantitative tests, such as topography and convergence. Validation of the statistical models used to assess topography are provided in Extended Data Figures 1-1, 1-2. These two metrics, topographic connectivity and convergence, have a negative nonlinear relationship (Extended Data Fig. 1-3). B, Time-series prediction model. For each time point, the overlap between the actual activation in the mapping region $[A(x, y, z, t)]$ and the fitted Gaussian distribution in the mapping region $\left[g_{v}(x, y, z)\right]$ for that particular seed voxel produce the expected level of activity in the corresponding seed voxel. The first arrow points to a period of low overlap, and thus a period of low expected level of activity, while the second arrow points to a period of high overlap, and thus a period of high expected level of activity. This procedure was used to evaluate the performance of the 3-D connective field model.

functional connectomes project (Biswal et al., 2010), and two smaller samples more well suited for test-retest reliability studies. The first small sample being the intrinsic brain activity test retest (IBATRT) dataset (Zuo et al., 2014), and the second being the midnight scan club (MSC) dataset (Gordon et al., 2017).

For the Cambridge Buckner data sample there were a total of 198 subjects (123 female), ages 18-30 $(\mathrm{M}=21.03, \mathrm{SD}=2.31)$, with all subjects included in the final analysis. For the IBATRT data sample, there were a total of 36 subjects (18 female) ages $19-48(M=27.33$, $\mathrm{SD}=7.86$ ) with two sessions and each with two runs. Four IBATRT subjects were excluded due to excessive motion in at least one of the four runs, leaving 32 subjects with data in the two runs of session 1 (16 female; ages $19-48, M=26.03, S D=7.24)$. For the MSC dataset, there were a total of 10 subjects (five female), ages 24$34(\mathrm{M}=29.1, \mathrm{SD}=3.3)$, and all included in the final analysis.

\section{fMRI parameters}

Cambridge Buckner data (Siemens 3T Trim Trio): T1weighted images were collected with MPRAGE with the following image parameters: slices $=192$, matrix size $=$ $144 \times 192$, voxel resolution $=1.20 \times 1.00 \times 1.33 \mathrm{~mm}^{3}$. Resting state fMRI data were $\mathrm{T}^{*}$-weighted images acquired using EPI with the following parameters: 47 interleaved axial slices, TR $=3000 \mathrm{~ms}$, voxel resolution $=3.0 \times 3.0 \times 3.0 \mathrm{~mm}^{3}$ (119 volumes).

IBATRT data (Siemens 3T Trim Trio): T1 weighted images were collected with MPRAGE with the following image parameters: slices $=176$, matrix size $=256 \times 256$, voxel resolution $=1.0 \times 1.0 \times 1.0 \mathrm{~mm}^{3}$. Resting state $\mathrm{fMRI}$ data were $\mathrm{T} 2^{*}$-weighted images acquired using EPI with the following parameters: 29 ascending axial slices, slice gap $=0.36 \mathrm{~mm}, \mathrm{TR}=1750 \mathrm{~ms}$, voxel resolution $=$ $3.4 \times 3.4 \times 3.6 \mathrm{~mm}^{3}$ (343 volumes each run). While there were up to four runs across two sessions for subjects, we only used the two runs in the first session.

MSC Data (Siemens 3T Trim Trio): Four T1 weighted images were collected: slices $=224$, voxel resolution $=0.8 \times$ $0.8 \times 0.8 \mathrm{~mm}^{3}$. Four T2 weighted images: 224 slices, voxel resolution $0.8 \times 0.8 \times 0.8 \mathrm{~mm}^{3}$. Resting state $\mathrm{fMRI}$ were T2*-weighted images acquired using EPI: 36 interleaved axial slices, TR $=2200 \mathrm{~ms}$, voxel resolution $=4 \times$ $4 \times 4 \mathrm{~mm}^{3}$ (818 volumes each session). A gradient echo field map was collected with the same parameters as the 
A
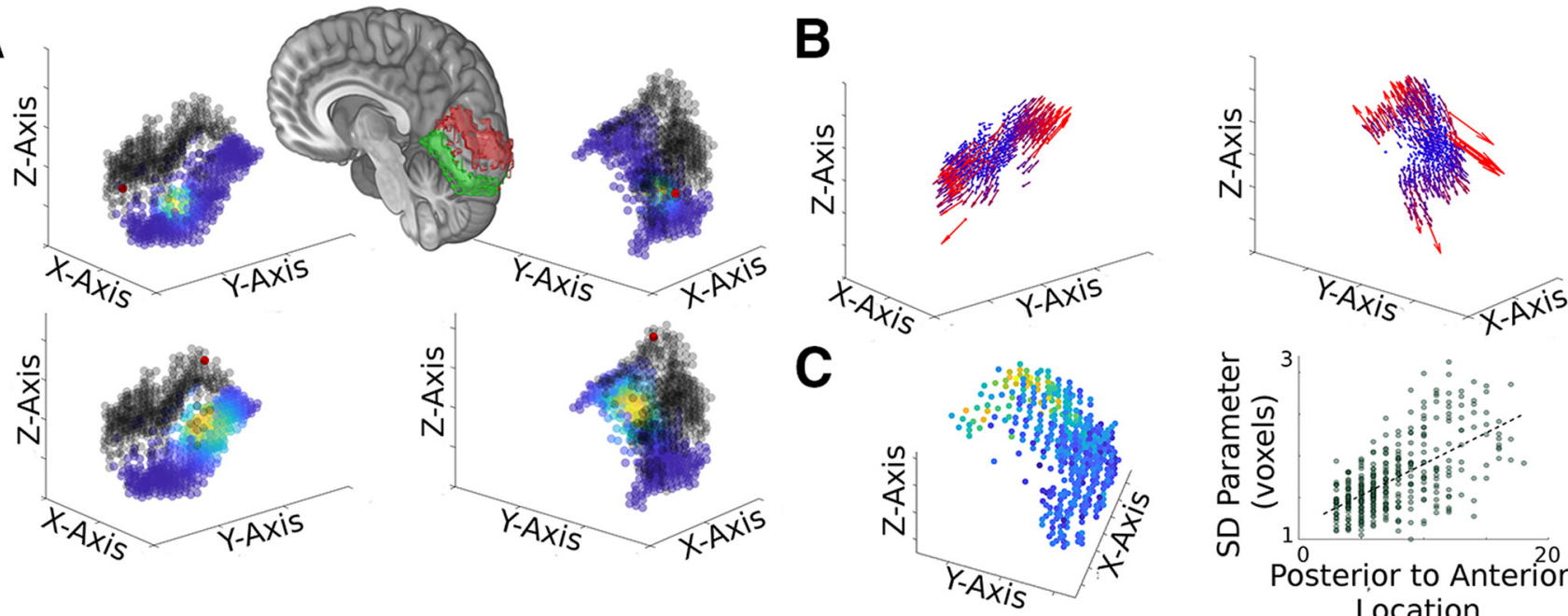

Location

Figure 2. Group results of the 3-D connective field modeling in dorsal and ventral visual cortex using the Cambridge Buckner resting-state fMRI dataset. $\boldsymbol{A}$, Examples of the resulting Gaussian model are shown for two seed voxels (red dots), plotted in red among other voxels (black circles) in dorsal visual cortex (red masked area in the right hemisphere), and their associated group level Gaussian fit (color scale ranged from blue to yellow) in ventral visual cortex (green masked area). For full visualization, each two column shows two plots of the seed voxel's fitted map rotated by $180^{\circ}$ from each other. $\boldsymbol{B}$, Preferred location of connectivity of each seed voxel is plotted as a vector with the origin in the seed location, and the vector direction showing the fitted $x_{0}, y_{0}$, and $z_{0}$ parameters. The vectors are colored coded with blue to red representing low to high Euclidean norm of the vector. While unclear in the current data, in higher resolution data, these vector fields are slightly deformed by the cortical surface (Extended Data Fig. 2-1). Extended Data Figure 2-2 shows the vector fields of dorsal and ventral visual cortex subregions. Further, we demonstrate the reliability of this result in Extended Data Figures 2-3, 2-4, 2-5. C, Group average of the Gaussian model's SD parameter. Left, Each voxel within the seed region, dorsal visual cortex, is plotted with the data point's color scaled by the SD parameter of their connective field model. Cooler to warmer colors represent smaller to larger SDs. Right, This plot shows the correlation between the anterior-posterior extent of the seed voxel and their associated SD parameter, suggesting that SD increases along more anterior voxels.

BOLD EPI images for each session. There were 10 sessions for each subject, all used in the analysis.

\section{Image preprocessing}

For each individual in the Cambridge Buckner and IBATRT data samples, preprocessing was performed using SPM12 (http://www.fil.ion.ucl.ac.uk/spm/software/ spm12/). The functional images were first corrected for slice timing, and then realigned to the middle volume according to a six-parameter rigid body transformation. Structural images were coregistered with the mean functional image, segmented, and then normalized to the MNI template using both linear and nonlinear transformations. Functional images were normalized using the same parameters as the structural normalization.

Further preprocessing was performed following the standard procedures of resting-state fMRI analysis either using CONN (Whitfield-Gabrieli and Nieto-Castanon, 2012) or custom MATLAB (2015b) scripts. A nuisance regression was constructed with the following confounding variables: six motion parameters up to their second derivatives, scans with evidence of excessive motion [framewise displacement (FD) $>0.5$ or DVARS $>5$ ], session onset, estimated physiological signal generated through aCompCor (a temporal PCA of the white matter and CSF voxels with the number of components included determined individually on the basis of a Monte Carlo null model; Behzadi et al., 2007), and a linear drift component.
For the Cambridge Buckner data the residuals from the nuisance regression were filtered using a bandpass between the frequencies of 0.008 and $0.09 \mathrm{~Hz}$, while for the IBATRT data, the bandpass filtering and nuisance regression were done simultaneously (Hallquist et al., 2013). Finally, the resultant data were despiked using a tangent squashing function.

For the MSC data, we used their release of preprocessed data which includes slice intensity correction, mode 1000 intensity normalization, realignment, transformation into Talairach space and FSL's field distortion correction (Smith et al., 2004). These data also came with additional preprocessing for resting-state analysis. Censored volumes were determined by an FD threshold of $0.2 \mathrm{~mm}$. The data were first demeaned and detrended, then a nuisance regression was performed removing the following factors, with censored volumes ignored: global signal, white matter mean signal, CSF mean signal, and motion regressors with the full Volterra expansion. The data were then interpolated across the censored volumes using least squares spectral estimation followed by bandpass filtering between 0.009 and $0.08 \mathrm{~Hz}$. The censored volumes were removed in the final resultant data samples for analysis (for full description of processing pipeline, see Gordon et al., 2017).

\section{Regions of interest (ROIs)}

The initial analyses of 3-D connective field mapping were conducted using the visual areas as ROls. For the 
early visual areas, we used the probabilistic visual atlas (Wang et al., 2015), selecting all voxels with any probability of either dorsal or ventral visual cortex, with no overlap between the two ROls (Fig. 2A). To determine whether the finding from the overall visual cortex mask was a general property of early visual areas, we also defined more selective masks of the dorsal and ventral portions of $\mathrm{V} 1, \mathrm{~V} 2$, and $V 3$, each with probability $>30 \%$ to reduce overlap between the masks. We then applied the same connective field mapping analyses across each visual region's dorsal and ventral portions.

In an additional analysis, we created slightly more lenient $\mathrm{V} 1$ mask selecting any voxels that have $\mathrm{a}>15 \%$ probability of being $\mathrm{V} 1$. We used this $\mathrm{V} 1 \mathrm{RO}$ as a mapping region for the right fusiform face area (FFA) and the right parahippocampal place area (PPA), both FFA and PPA were derived using Neurosynth meta-analyses, with "faces" and "place" as keywords, respectively (Yarkoni et al., 2011).

For the whole-brain topographic connectivity analysis, we made use of a previously published whole-brain parcellation atlas of 400 regions. The parcels were generated by maximizing correlations within a parcel while minimizing local changes in correlations within a parcel (Schaefer et al., 2018). For time and computational feasibility, we only used the 200 parcels of the right hemisphere.

\section{3-D connective field mapping and model parameters}

The 3-D connective field mapping analysis has been released in the form of a MATLAB toolbox, with a simple GUI wrapper to help guide analysis (www.nitrc.org/ projects/r2r_prf/).

For both the "seed" and "mapping" regions, the mean time course of the ROI was subtracted from each voxel's time course, so as to reduce the influence of correlation at the mean signal level, although in practice the results are similar with or without removing the means. Then, for each voxel within the seed region, we calculated its correlation with every voxel within the mapping region and transformed the resulting correlation values using Fisher's $Z$ transformation. Each distribution of Fisher's $z$ values (for each seed voxel) was fitted with a 3-D isotropic Gaussian distribution (Fig. 1A):

$$
\begin{aligned}
g_{v}(x, y, z)= & \frac{1}{\sigma^{3}(2 \pi)^{3 / 2}} \exp \\
& -\frac{\left(x-x_{0}\right)^{2}+\left(y-y_{0}\right)^{2}+\left(z-z_{0}\right)^{2}}{2 \sigma^{2}}
\end{aligned}
$$

Initial values for the $x_{0}, y_{0}$, and $z_{0}$ parameters during the optimization procedure were the $x-, y-$, and $z$-locations of the maximum Fisher's $z$ value, and an arbitrarily small number (floating-point epsilon) for the SD. The optimization procedure had lower bounds of the smallest location parameters within the mapping region and the floatingpoint epsilon for the SD, while the upper bounds were the maximum location parameters and the smallest range of the three dimensions for the SD. The parameters of the
Gaussian were estimated by minimizing the correlation distance between the Fisher's $z$ values and the Gaussian probability density function using the interior-point algorithm (Byrd et al., 2000), implemented by MATLAB's fmincon function (MathWorks Inc, 1995-2019). Predicted time series for each voxel $v$ in the seed region were estimated as the degree of overlap between the estimated Gaussian function and the activation pattern of the mapping region for each time point $t$ (Fig. 1B):

$$
p_{v}(t)=\sum_{x} \sum_{y} \sum_{z} A(x, y, z, t) g_{v}(x, y, z)
$$

Both the predicted time series and the actual data time series were z-scored to remove any arbitrary scaling factors. Fit was assessed by comparing the distribution of correlations between each voxel's fitted model and the true data to the distribution of correlation between each voxel's true data and every other voxel's fitted model.

\section{Metrics of region-to-region organizational patterns}

Topographic organization of functional connectivity between the seed and mapping region was estimated as rank linearity: estimated using the Procrustes method by fitting the three rank-ordered space parameters of the Gaussian model to the three rank-ordered space parameters of the seed locations (Kendall, 1989). The 3-D estimated parameters from the 3-D connective field model where first rank ordered, and then a transformation matrix with translation, rotation, and scale components was estimated that minimizes the normalized sums of squares error (SSE) between the rank ordered parameters and their spatial origin. The complement of the normalized SSE between the transformed parameters and the actual seed locations was used as a measure of the degree of topographic connectivity between two regions.

In order to determine whether the topographic arrangement of a region's functional connectivity is above and beyond the base topography expected due to chance (given BOLD data characteristics), we formulated a way to examine what it means for a region to be topographically organized. Given the probability of a voxel (in a volume of randomly distributed data) having a data rank order equal to its spatial rank $[p(r)]$ is the inverse of the volume $(\mathrm{V})$ of the seed:

$$
p(r)=\frac{1}{V}
$$

Then, the expected rank linearity of a volume is the average probability of this occurrence across the volume, which is simply the inverse of the volume of the seed again:

$$
E(\operatorname{Lin})=\frac{\sum p(r)}{V}=\frac{\sum \frac{1}{V}}{V}=\frac{1}{V}
$$

then, understanding the effect of smoothing on this expected value is understanding the extent to which smoothing reduces the number of independent 
observations in the spatial field, as defined by the number of resels in the volume:

$$
E\left(\text { Lin }_{\text {smoothness }}\right)=\frac{1}{V / F W H M^{D}}
$$

where FWHM is the estimated smoothness of the volume, and $D$ is the dimensionality of the data (three in the case of $\mathrm{fMRI}$ data). Statistical inferences can be made by evaluating the $95 \%$ confidence intervals of this probability on a binomial distribution given the number of voxels in the volume.

To examine whether the process described reflects the behavior of our rank linearity estimations, we simulated volumes of randomly distributed data while manipulating both the number of voxels in the volume and the smoothness. First, we showed that the expected value of rank linearity is approximately equal to the probability of a rank, which is the inverse of the voxels in a volume (Extended Data Fig. 1$1 A)$. Second, we found that the effect of smoothing follows from the approximate number of independent observations (i.e., number of resels) within the volume (with the 95\% confidence intervals well approximated by evaluation on the binomial; Extended Data Fig. 1-1B). However, we noted that there seemed to be some divergence of the model from the simulated data at higher smoothing values. We then conducted more simulations to test whether the deviance was related to the total number of independent observations in the volume, by manipulating the number of voxels in the volume. If the deviance is due to the total number of independent observations (resels) then this data should diverge from the model with a lower smoothing FWHM in simulations with fewer voxels. We found that this was roughly the case [Extended Data Fig. $1-2 A$ (volume $=729$ voxels) vs Extended Data Fig. 1$2 B$ (volume $=125$ voxels)]. We selected a rule of thumb to flag inferences that may be inappropriate, where the number of resels in a ROI is less than or equal to 10 .

In addition to topographic connectivity, we also estimated convergence of functional connectivity. Convergence is the logical counterpart to topographic connectivity, and was calculated using a 3-D form of the Kolmogorov-Smirnov (KS) test (Fasano and Franceschini, 1987). The convergence metric was defined as the deviance of the Gaussian parameters from uniform distribution across the voxels in the mapping region. This provides a relatively distribution insensitive measure of convergence (Fasano and Franceschini, 1987). To correct for regional differences in spatial correlations and number of voxels, we used the KS value corrected for the KS value at the $5 \% \alpha$ level. The relationship between convergence factor and rank linearity is shown in Extended Data Figure 1-3.

Further, vector fields were constructed to visualize the connectivity organization between two regions, with each vector originating at each seed voxel, and the orientation and length of the vector determined by the centered Gaussian parameters, its preferred location in the mapping region in relation to the center of mass of the mapping region (Fig. 2B).

However, corticocortical relationships are likely to be obfuscated in 3-D space due to cortical folding. While previous attempts to examine this have aligned data to the cortical surface to avoid this problem, we did not use cortical sheets for several reasons. First and foremost, since the data are acquired in volumetric space, most operations aligning to a cortical surface model results in additional smoothing to the data (e.g., through averaging). In addition, unfolding the cortical sheet is an arbitrary process, with distortions added in different forms depending how it is done. Therefore, constructing objective 2-D coordinates is intractable. Our measures of topography provided novel information in the original space, but are likely influenced by the different folding patterns of the cortex to some extent. Therefore, we also visualize the relationship between regions by constructing a vector field. The vector field allows the researcher to observe when spatial relationships from one cortical region conform to the cortical surface, as the vector field should bend along sulci and gyri (for an example in a single subject, and group vector field on how vectors are distorted when constrained to the cortical sheet, see Extended Data Fig. 2-1).

We assessed test-retest reliability using the IBATRT and MSC datasets. For the IBATRT dataset, we examined the spatial correlation between the patterns of parameters in run 1 and run 2, within individual subjects and across session means. For the MSC dataset, we examined potential effects of added scan time to the stability of the 3-D. Gaussian parameters for individual subjects. We split each subjects' sessions into two groups, a test and a retest group. We varied the number of sessions in each group, with a maximum of 5 session per group, and calculated the test-retest reliability for each subject. We also performed 3-D connective field mapping for each subject with all their sessions to demonstrate the stability of the group effect at the level of individual subjects.

\section{Examining connectivity profiles in higher order visual regions}

To study how the patterns of connectivity from early visual cortex to higher order visual regions, such as FFA and PPA (for ROI selection, see above, Regions of interest), may predict their complex response properties, we fit 3-D connective field models seeding from these areas, mapping into primary visual cortex. We compared convergence and topographic connectivity between PPA/FFA and V1, and compared the model parameters to those of the intrinsic convergence and topographic connectivity within V1.

We expected both regions to have higher convergence than the relationships between dorsal and ventral V1. Given the observed retinotopy in PPA (Arcaro et al., 2009), and the relatively weaker observed retinotopy in FFA (Saygin and Sereno, 2008), we also expected PPA to demonstrate a higher amount of topographic connectivity and a lower amount of convergence with $\mathrm{V} 1$ relative to FFA connectivity relationships with $\mathrm{V} 1$.

\section{Whole-brain characterization of topographic functional connectivity}

We performed 3-D connective field mapping on each unique pair between all possible pairs out of the 200 
parcels in the right hemisphere of the Shaefer atlas for each subject in the MSC data. For each fitted pair, we calculated the rank linearity of connectivity using Procrustes analysis. We selected several seed regions to test whether topography is maintained through specific networks or generally across the brain. We selected several a priori networks and examined whether their topographic characteristics follow network like behavior, namely that their within-network topographic connectivity is stronger than between network topographic connectivity.

As a higher order summary, and to compare with previous whole-brain gradient decompositions (Margulies et al., 2016; Murray et al., 2018), we performed nonmetric multidimensional scaling on the rank linearity matrices from all parcel pairs to estimate the gradients of topographic connectivity across the right hemisphere. The far ends of these gradients were considered to represent which regions maintain linear information across the brain. We produced a two mode solution, on the basis of an elbow in the plot of the rank correlation of the produced distance and the observed distance matrices (Extended Data Fig. 6-1A), and visualized the gradients across the brain.

\section{Lateral prefrontal cortex analysis}

We selected the subset of parcels (see above, Regions of interest) within the lateral prefrontal cortex, and performed a similar gradient decomposition analysis specifically on prefrontal functional connectivity topography. We estimated two gradients (for rational, see Extended Data Fig. 6-1B) of the lateral prefrontal cortex and then examined whether the discovered organization reflects principles of prefrontal cortex organization described in the literature (Goldman-Rakic, 1987; Koechlin et al., 2003). For comparison, we further examined the gradients resulting voxel-wise functional connectivity (Extended Data Fig. 7-1).

Each participant of the MSC data sample also went through three tasks in the scanner, an incidental memory task in which participants judged various characteristics of faces, scenes, and words, a motor task in which participants were instructed to move various parts of their body, and a mixed block task design in which participants had to either discriminate between nouns and verbs, or coherently and incoherently moving dots, with a cue on the onset and offset of each block (Gordon et al., 2017). We used two contrasts to examine sensory specialization (memory: face - scene, mixed: visual - semantic discrimination) and one to examine abstract rules [mixed: discrimination (visual and semantic) - cue effects].

In order to examine how the organizational features of the lateral prefrontal cortex observed in the resting state scans relate to spatial patterns of task activation, we constructed three multiple regressions, one for each contrast, predicting task activation from each topographic gradient and average convergence.

\section{Results}

\section{Mapping functional connectivity in the visual cortex}

To replicate previous findings from 2-D modeling on the surface (Haak et al., 2013; Gravel et al., 2014), we first used 3-D connective field modeling to show that dorsal and ventral portions of the visual cortex have a topographic organization in their functional connectivity. Figure $2 A$ illustrates the group average 3-D connective field maps in the ventral visual cortex for two selected seed voxels in the dorsal visual cortex using the Cambridge Buckner dataset. Figure $2 B$ summarizes these results as a vector field in the seed region, with a vector originated from each seed voxel displaying the location of its preferred connectivity as the relative displacement from the center of the mapping region (Fig. 2B). This vector field of the dorsal visual cortex revealed a strong connectivity mapping to the ventral visual cortex along the predicted anterior-posterior organization (Arcaro et al., 2015), which was also supported quantitatively by the high correlation between the seed's $y$-location and the mean modeled $y$-location of the Gaussian fit in the mapping region, with $r_{(394)}=0.71, p=1.63 \times 10^{-62}(r=0.83$, with outliers removed). The spread or SD parameter of the Gaussian model also increased along the seed region's $y$-axis (Fig. 2C, left), which was supported quantitatively by the correlation between the seed's $y$-location and the modeled SD parameter, with $r_{(394)}=0.61, p=$ $5.45 \times 10^{-42}$ (Fig. $2 C$, right). This relationship was similarly evident between the dorsal and ventral portions of $\mathrm{V} 1, \mathrm{~V} 1$, and $\mathrm{V} 3$, using a set of more restricted masks (Extended Data Fig. 2-2). These results together suggested that the inter-regional connectivity in early visual areas mirror the organization of eccentricity representation, with region-to-region connectivity ordered spatially along similar eccentricity locations, and the spread of that connectivity potentially following the increase in receptive field sizes toward more anterior visual cortex. While the group averaged data can provide qualitative aspects of the organization of regions, the averaging procedure can introduce various biases (and average out individual differences to produce an optimistically simple output).

To quantify the organization at an individual subject level, we first quantified whether or not these measures are stable at the individual subject level data, and further we moved to a data sample that has much longer BOLD collections to ensure high-quality estimations (MSC). The median test-retest reliability within subjects increases with more scan time per subject, with the 150 min in each split leading to a test-retest reliability of 0.81 (Extended Data Fig. 2-4C). A vector field of dorsal-ventral connectivity mapping for the visual cortex was generated for each individual and each session, and the anterior-posterior relationship was observed in every subject (Extended Data Fig. 2-5). Quantitatively, the correlation between $y$-location modeled Gaussian parameter and seed $y$-location was high: ranging from 0.5 to 0.72 across the 10 individuals. In addition, topographic connectivity estimates for the entire dorsal and ventral visual cortex for each MSC individual ranged between $13.77 \%$ and $18.27 \%$ (all $\left.p_{\text {FWE' }}<0.05\right)$. Similarly, topographic connectivity estimates of $\mathrm{V} 1 \mathrm{~d} \rightarrow \mathrm{V} 1 \mathrm{v}$ were highly replicated in each MSC subject, ranging from $24.62 \%$ to $34.71 \%$ (only one session of MSC02 and one session of MSC08 $>\alpha_{\mathrm{FWE}}$ ). 


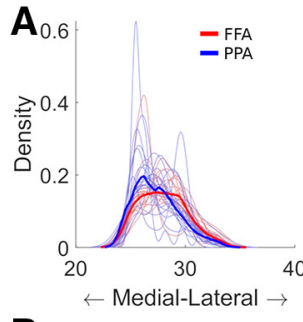

B

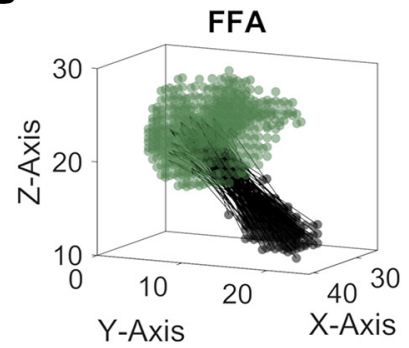

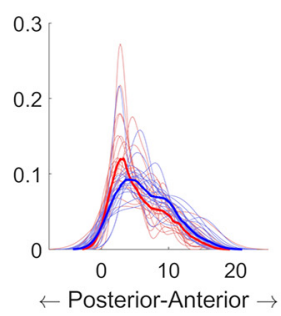

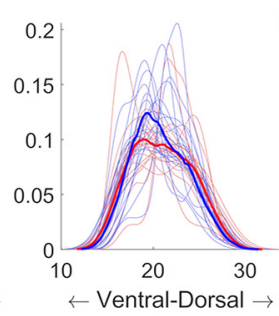

PPA

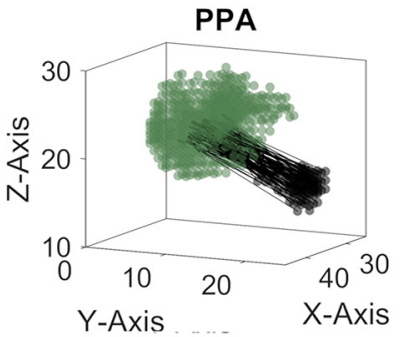

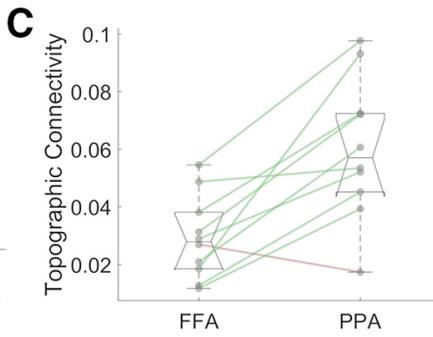
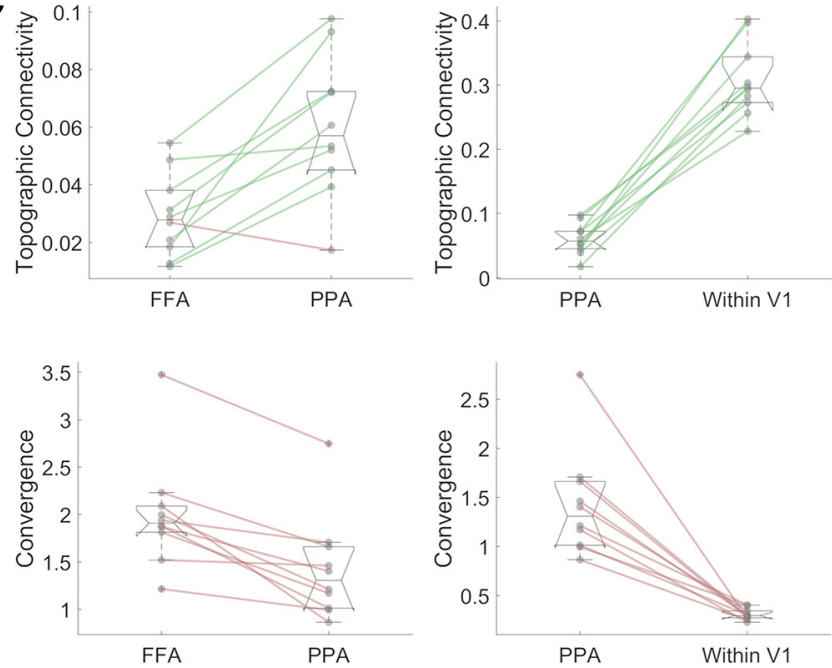

Figure 3. Pattern of functional connectivity between higher order visual regions and $\mathrm{V} 1$ corresponds well with their visual response properties from previous studies. A, Compared with the PPA, FFA showed a more posteriorly located and narrower range of connectivity with V1. Thin lines represent kernel density plots of individual subjects of the MSC dataset, while thick lines represent the kernel density plot of the group average data. $\boldsymbol{B}$, Another representation of the biases of FFA to V1 connectivity (left) in comparison to PPA to V1 connectivity (right). The black points represent voxels within the seed region and the green points represent voxels within the mapping region, with the vectors connecting them represent the seed voxels' preferred connectivity location along the mapping region. The mean vector fields provide information about quantitative features of the connectivity (such as the relatively more focal and mixing connectivity seen in FFA vs PPA) but to quantify these results requires computation on individual subject vector fields. $\boldsymbol{C}$, Estimations of topographic connectivity show higher topography with V1 maintained in PPA, and higher convergence with V1 in FFA. Both of these metrics are shown next to dorsal and ventral V1 (within V1) estimates for reference.

We also evaluated the model validity and stability in several ways. First, we examined the distribution of correlations between the predicted time courses from the model and the actual BOLD time courses (Fig. 1B) for each voxel in the large dorsal visual cortex mask (Extended Data Fig. 2-3, left). The distribution of $r$ values across all subjects and all their correspondent time courses when using the matching prediction was strictly positive, with a mean of 0.55 , whereas the distribution of correlations produced by nonmatched pairs is much wider with a mean of close to zero (Extended Data Fig. 23 , right). Second, also evaluated the stability of the 3-D connective field model within individual subjects and across mean results for the dorsal and ventral visual cortex, using the IBATRT dataset. For each subject, the estimated $y$-location parameters from the first and second runs were correlated across voxels (Extended Data Fig. 2$4 A$ ). The resulting distribution of $r$ values had a median of 0.60 , demonstrating moderate to good reliability at the individual subject level. At the group level, the mean parameters for each run correlated across voxels strongly, with $r=0.98$ (Extended Data Fig. 2-4B).

\section{Connectivity bias in higher-order visual areas}

Higher order visual regions have been characterized as complex filters of early visual region information (Van Essen and Gallant, 1994), with this type of model shown to predict FFA activation during a task (Kay and Yeatman, 2017). We thus examined to what extent the pattern of region-to-region functional connectivity reflect this filtering process. Using the MSC data, we applied the 3-D connective field mapping method to model the pattern of right FFA connectivity with $\mathrm{V} 1$ and compared its connectivity profile with that of PPA, a region which is selective to stimuli that have more peripherally distinctive features. Because of initial poor $r^{2}$ of the Gaussian fits, we combined the sessions into two distinct sessions, allowing for better estimation across each. In comparison to the PPA, the FFA showed more connectivity with posterior and lateral V1 $\left(t_{(9)}=-7.04, p=6.04 \times 10^{-5}\right.$ and $t_{(9)}=3.36$, $p=0.008$, respectively; Fig. $3 A$ ). This is consistent the FFA receiving information from $\mathrm{V} 1$ areas with more foveal receptive fields. For visualization, we plotted group averaged vectors from each seed region to V1. From these vector fields, FFA seems to share more convergent and less topographic connectivity with V1 (Fig. 3B). We quantified this in individual subjects and compared across regions, with V1d to V1v connectivity for reference. When compared with PPA, the FFA has both significantly higher convergence and significantly lower topographic connectivity with V1 $\left(t_{(9)}=5.25, p=5.27 \times 10^{-4}\right.$ and $t_{(9)}=-4.33$, $p<0.002$, respectively; Fig. $3 C$ ). PPA, when compared with measures within $\mathrm{V} 1$, in turn, shows lower linearity and higher convergence.

\section{Topographic connectivity organization across the cortex}

While the early visual system demonstrated large topographic relationships in functional connectivity between regions, a fundamental question left unanswered is whether this is a special property of the early visual cortex or it is a general motif of corticocortical functional 


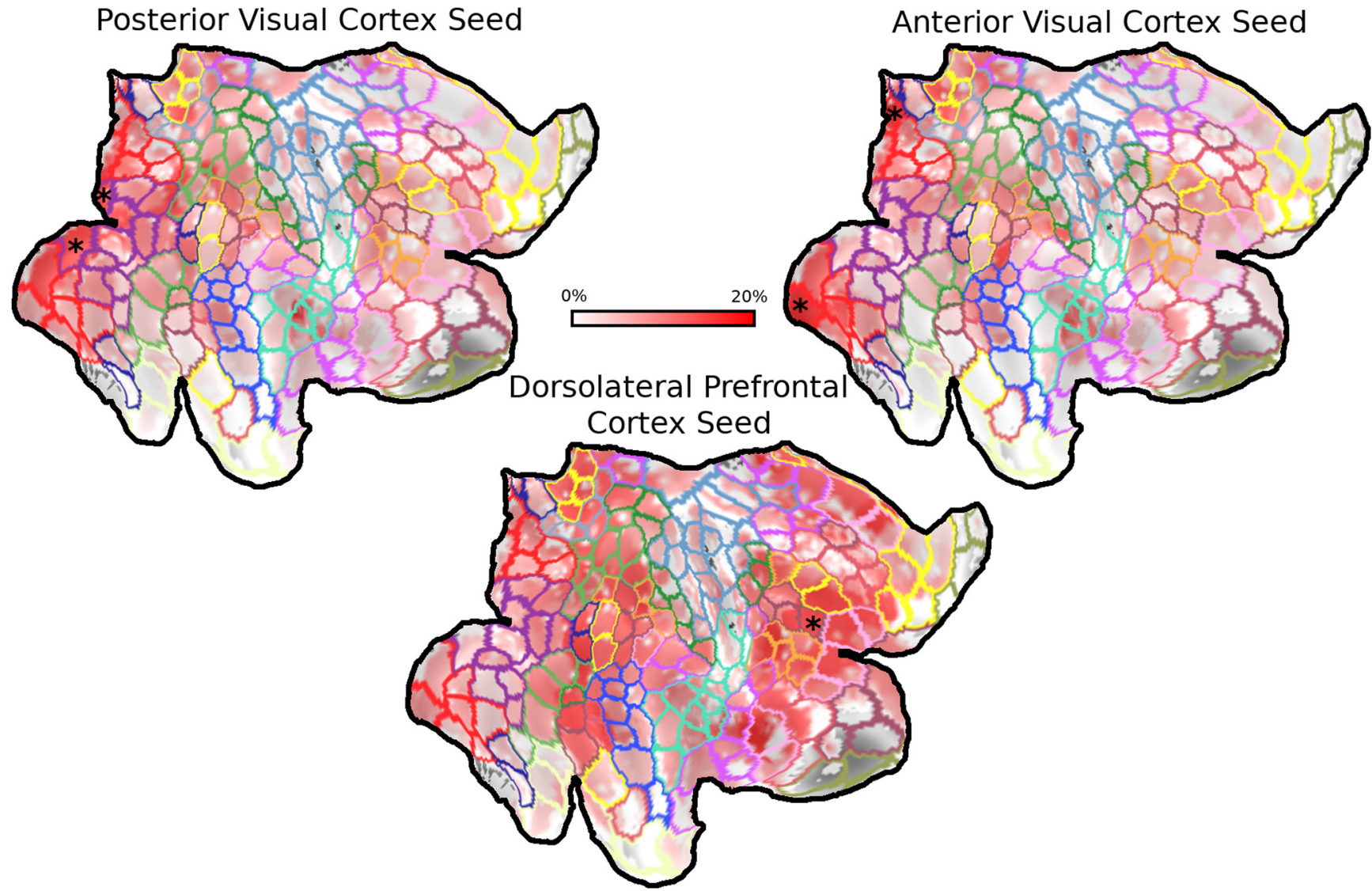

Figure 4. Topographic connectivity as a general rule of functional connectivity between cortical regions. Topographic connectivity estimates are shown from three seed regions (shown as asterisk in each map), posterior visual cortex, anterior visual cortex, and dorsolateral prefrontal cortex. The color scale from darker to brighter color represents lower to higher topographic connectivity for the seed regions' connective field in the other brain regions. This whole-brain illustration demonstrates that while topographic connectivity is a general phenomenon for region-to-region connectivity, it is maintained within specific networks, suggesting function in maintaining integrity of information transfer across regions within a network. Extended Data Figure 4-1 shows each of these seeded maps in a single subject of the MSC data.

connectivity. To address this issue, we calculated the linearity of connectivity between all unique pairs of the 200 right hemisphere parcels in the Schaefer parcellation. We used the MSC dataset for this analysis to ensure stable parameter estimates, given the long scan time for each session. For each subject of the MSC dataset, the average percentage of connections that surpassed $\alpha=0.05$ threshold across all sessions and all pairs of parcels was $20.60 \%$ (range across subjects: $13.30-25.69 \%$ ). Note that the subject with the lowest number of significant topographic relationships, MSC08 (with $13.30 \%$ of connections surpassing $0.05 \alpha$ ), was identified by the original investigators to have a significant proportion of time in a drowsy state, affecting this individual's BOLD data (Gordon et al., 2017). Therefore, on average, each brain region maintains a detectable topographic relationship with $\sim 20 \%$ of the rest of the brain, at a liberal threshold for detection. Eliminating parcels which likely do not follow the assumptions of our model (due to low number of resels) increases this average percentage (to $23.54 \%$, ranging from $14.25 \%$ to $29.64 \%$ ).

Figure 4 illustrates the linear organization for three selected seed regions' connectivity with other regions across the entire right hemisphere. The flatmaps show a clear segregation of linearity values within and across the different networks, exemplified by seeding from anterior and posterior visual cortex demonstrating stronger linearity with other visual areas, middle frontal gyrus demonstrating stronger linearity with lateral prefrontal and parietal regions, and lower linearity between regions from different networks. In Extended Data Figure 4-1, we present MSC01's session by session seed rank linearity maps, presented with $-\log (p)$ values to demonstrate the stability of the statistical inference.

To visualize and quantify the network basis of topographic organization of region-to-region connectivity, we constructed a graph representation of our rank linearity maps (Fig. 5A). We then quantified within and between network linearity for each of the $10 \mathrm{MSC}$ subjects across three different networks (VisCent, VentralAttenB, DorsalAttenB). For each of the six possible comparisons, the within network rank linearity was significantly higher than the between network rank linearity $\left(t_{(9)}=9.52, t_{(9)}=3.80, t_{(9)}=9.26, t_{(9)}=3.19\right.$, $t_{(9)}=9.75$, and $t_{(9)}=6.75 ; p=5.38 \times 10^{-6}, p=4.00 \times 10^{-3}$, $p=6.76 \times 10^{-6}, p=1.10 \times 10^{-2}, p=4.40 \times 10^{-6}$, and $p=8.33 \times 10^{-5}$; Fig. $5 B$ ). 

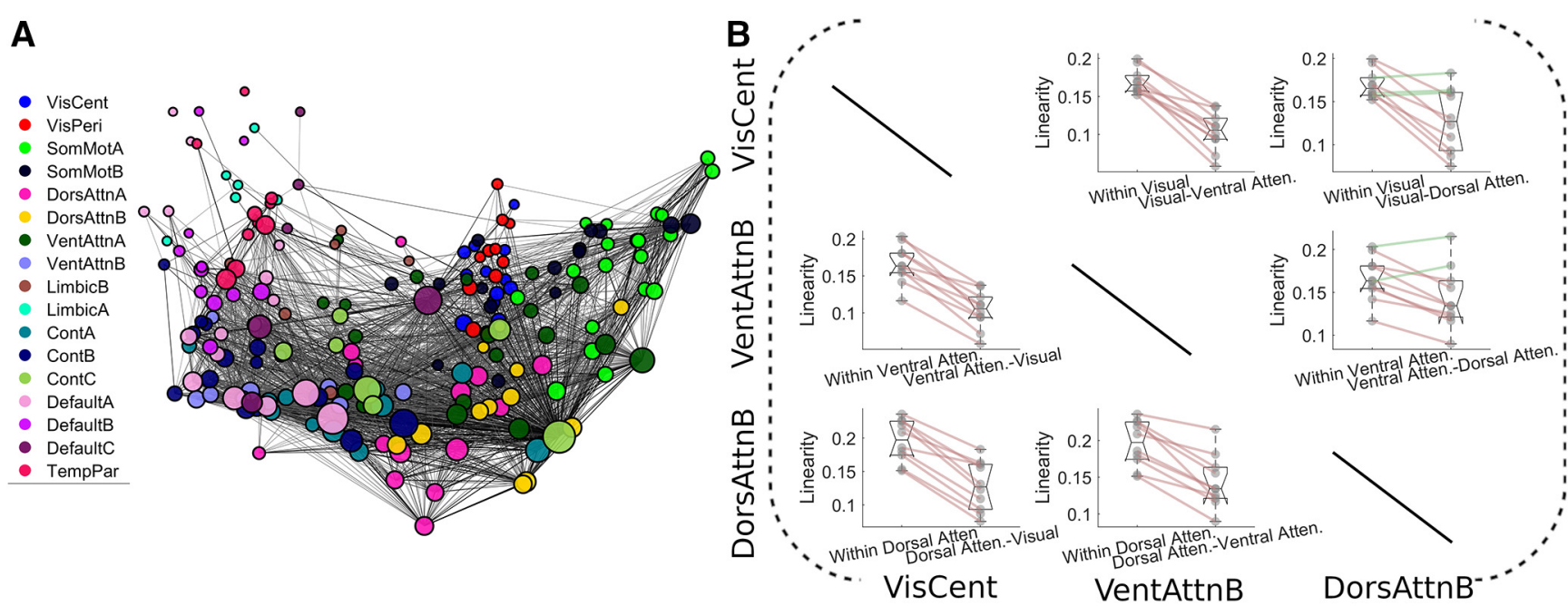

Figure 5. Network analysis. A, Graph description of our topographic network analysis using the 17 network labels from the Schaefer atlas. Node position was determined by performing classical multidimensional scaling on the full linearity matrix, and then an edge was drawn if it surpassed a linearity threshold of $20 \%$. Node size was scaled linearity in relation to the node's degree in the thresholded network. B, Quantification of the network like organization of linearity with three example networks, visual (central), ventral attention B, and dorsal attention B. The within network linearity is universally higher than the between network linearity in these three networks, as can be seen in $\boldsymbol{A}$ by the relative closeness of nodes within a network. Note: Schaefer atlas used for network labels (for more details, see Schaefer et al., 2018).

To examine whether there are any higher order (supranetwork) organizational features of topographic connectivity, nonmetric multidimensional scaling was applied to produce two dimensions that maximally reproduce the nonlinearity matrix (as a distance matrix) the 200 parcels in the right hemisphere (Extended Data Fig. 6-1; Fig. 6).

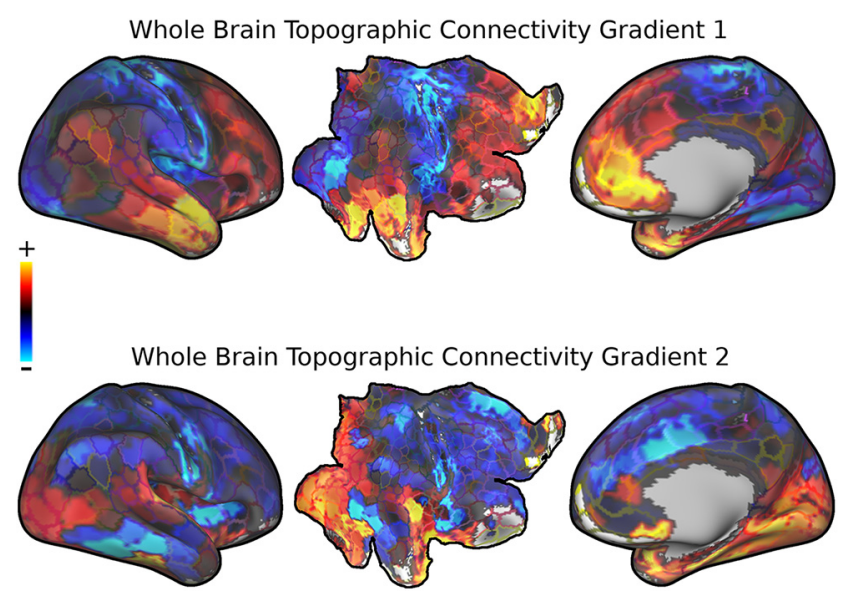

Figure 6. Gradient decomposition of whole-brain linearity (for rational for number of estimated gradients, see Extended Data Fig. $6-1)$. Whole-brain region-to-region linearity matrix was decomposed using nonmetric multidimensional scaling in order to examine organizational patterns of topographic connectivity across the brain. The gradients are color scaled from red to blue, with red-yellow colors representing one side of the gradient, and blue-green colors representing the other side of the gradient. Top, First whole-brain linearity gradient, demonstrates a segregation of unimodal sensory areas and multimodal association areas. Bottom, Second whole-brain linearity gradient, the fractionated nature suggests potential integration of information across unimodal sensory areas and multimodal association areas.
The resulting primary mode was similar to large scale whole-brain decompositions observed in previous studies of normal functional connectivity and genetic expression data (Margulies et al., 2016; Murray et al., 2018). This mode described a segregation lower order unimodal regions and higher order multimodal regions, with the negative end exemplified by visual and somatomotor networks and the positive end exemplified by default mode network. The second mode seemed to be a fractionation of the primary mode, potentially representing communication of information across these lower order/higher order boundaries.

\section{Connectivity organization of the lateral prefrontal cortex}

After illustrating the ubiquity of topographic organization of functional connectivity across visual cortex and large networks, we specifically examined how gradients of linearity express in a highly multimodal structure such as the lateral prefrontal cortex. We found two gradients (Extended Data Fig. 6-1B). Gradient 1 demonstrates a rostral-caudal axis that segregates posterior middle frontal gyrus/inferior precentral sulcus and anterior inferior frontal gyrus/frontal pole (Fig. 7A, top left). Gradient 2 demonstrates a diagonal axis, segregating posterior superior frontal gyrus and anterior inferior frontal gyrus (Fig. 7A, top right). These two topographic connectivity gradients closely resemble the two prominent frameworks described in the literature to simplify the overall functional organization of the frontal cortex. One is often referred to as the domain specialization framework, suggesting that the dorsal and ventral lateral prefrontal cortices are preferentially organized to process spatial and nonspatial (e.g., objects) information, respectively, due to their differential connectivity with the dorsal and ventral visual streams (Goldman-Rakic, 1987). The second is an 

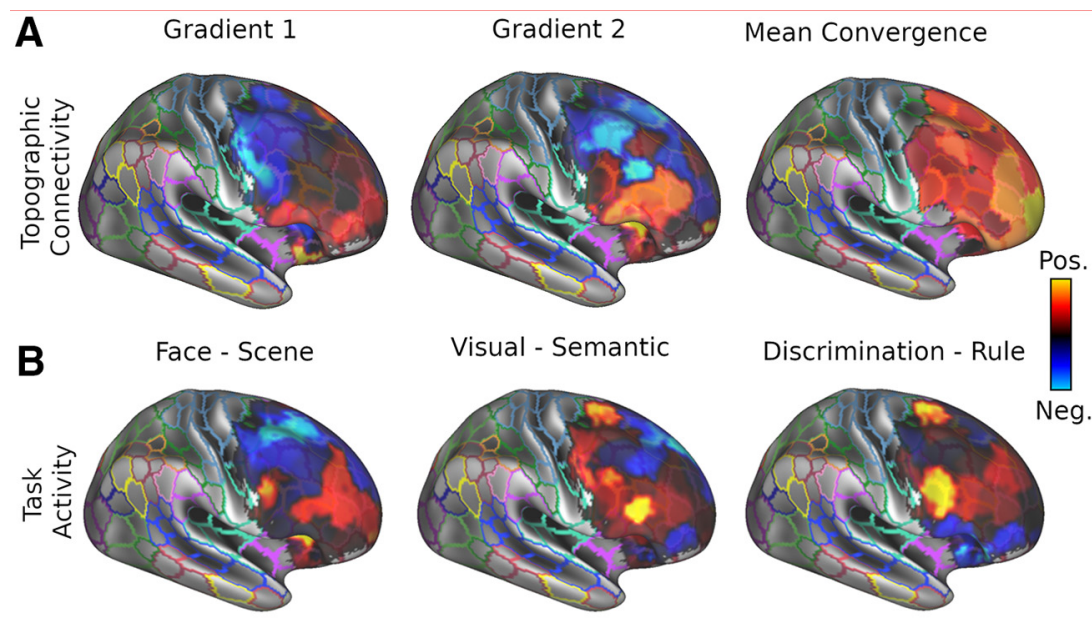

Discrimination - Rule
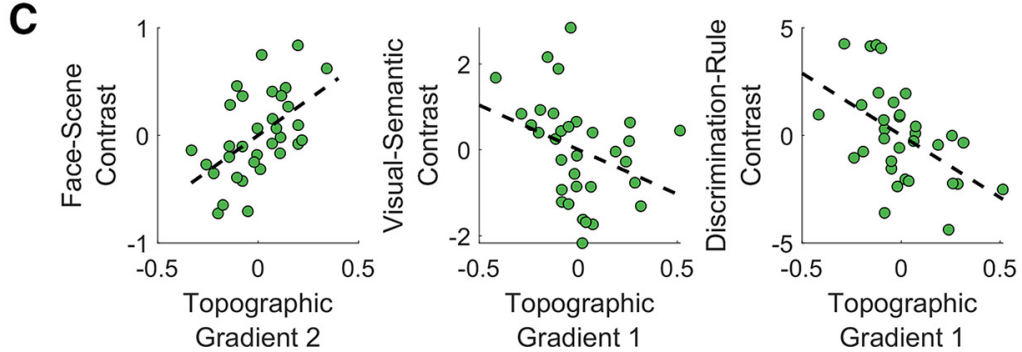

Figure 7. Patterns of functional connectivity within lateral prefrontal cortex. $\boldsymbol{A}$, The decomposition of the bivariate rank-linearity of the lateral prefrontal cortex revealed two gradients. Gradient 1 demonstrates an organization from posterior middle frontal gyrus/inferior precentral sulcus to anterior inferior frontal gyrus/frontopolar cortex, while gradient 2 demonstrates an organization from posterior superior frontal cortex to anterior inferior frontal gyrus. The average convergence factor demonstrates a peak along the frontal pole (for similar gradient decomposition of voxel-wise functional connectivity, see Extended Data Fig. 7-1). B, Three different contrasts were examined to correlate to frontal organizational features: face-scene to examine visual specialization, visual-semantic discrimination to examine sensory specialization, and discrimination-rule to examine levels of abstraction. $\boldsymbol{C}$, First-order correlation demonstrating the most highly associated organizational feature with each contrast (for full regression analysis results, see Table 1).

increase in abstraction along the rostral-caudal axis, with the more rostral regions linked to more abstract facets of behavioral control (e.g., sensory perception $\rightarrow$ context $\rightarrow$ rule; Koechlin et al., 2003). In addition, we performed nonmentric MDS on the standard voxel-wise resting-state functional connectivity of the lateral prefrontal cortex, and found two gradient decompositions that mainly segregate middle frontal gyrus and inferior frontal gyrus from the rest of the frontal cortex (Extended Data Fig. 7-1).

We examined whether the average task activation patterns for sensory specialization and level of abstraction from the MSC data sample (Fig. 7B) can be predicted by these organizational features. We found that a linear model with each topographic gradient and convergence predicts each of the activation patterns from each of the contrasts (facescene: $R^{2}=0.37, p=0.0030 .01$; visual-semantic: $R^{2}=0.24$, $p=0.046$; discrimination-rule: $\left.R^{2}=0.41, p=0.002\right)$. The strongest predictor of face-scene contrast was the gradient 2 value $\left(\beta=0.55, p=8.74 \times 10^{-4}\right)$, while the strongest predictor for the discrimination-rule contrast was the gradient 1 value $\left(\beta=-0.56, p=6.34 \times 10^{-4}\right.$; Fig. $7 C$; Table 1$)$. This supports the above interpretation of the two gradients of topographic connectivity as relating to domain specialization and abstraction, respectively.

\section{Discussion}

Using a 3-D Gaussian modeling approach with wholebrain resting-state fMRI data, we found that closely connected cortical regions exhibit a relatively strong topographic organization of their functional connectivity. This topographic organization of region-to-region functional connectivity was highly reliable in early visual regions, following an order along the anterior-posterior axis for eccentricity representation, as predicted by the literature (Haak et al., 2013; Gravel et al., 2014; Arcaro et al., 2015). In higher order visual regions, such as the FFA and PPA, the estimated connectivity profile seemed to reflect complex filtering of information, such that FFA connectivity to $\mathrm{V} 1$ produced the hypothesized patterns of bias towards the part of $\mathrm{V} 1$ that has more foveal representations. Further, the PPA shows a more maintained topography with V1 in comparison to PPA. These results are consistent with previous attempts to compare these two higher order regions using a different multivariate method to estimate voxel level patterns of connectivity from one region to another (Baldassano et al., 2012), where it was found that FFA shows connectivity bias to more foveal resources, while PPA shows a connectivity bias to more eccentric resources. In the whole brain, topographic connectivity again 
Table 1: Organizational features of lateral frontal cortex relate to task activation

\begin{tabular}{|c|c|c|c|c|}
\hline \multicolumn{5}{|c|}{ Face-scene contrast (incidental memory t): $R^{2}=0.37$} \\
\hline & Beta & SE & $t$ value & $p$ value \\
\hline Gradient 1 & -0.06 & 0.15 & -0.41 & 0.6822 \\
\hline Convergence & 0.31 & 0.15 & 2.08 & 0.0469 \\
\hline \multicolumn{5}{|c|}{ Visual-semantic contrast (mixed task): $R^{2}=0.24$} \\
\hline & Beta & SE & $t$ value & $p$ value \\
\hline Gradient 2 & 0.26 & 0.16 & 1.60 & 0.1207 \\
\hline Convergence & 0.26 & 0.17 & 1.56 & 0.1293 \\
\hline \multicolumn{5}{|c|}{ Discrimination, rule contrast (mixed task): $R^{2}=0.41$} \\
\hline & Beta & SE & $t$ value & $p$ value \\
\hline Intercept & 0.00 & 0.14 & 0.00 & 1.0000 \\
\hline Gradient 1 & -0.56 & 0.15 & -3.83 & 0.0006 \\
\hline
\end{tabular}

emerged as a primary organizational feature for functional connectivity among regions within large-scale networks, including the default mode and frontoparietal networks, while such relationships were much weaker between regions across different networks. Lastly, we found that the topographic and convergence patterns of connectivity in the lateral prefrontal cortex supports the two prevailing frameworks of prefrontal cortex functional organization, domain specialization and abstraction. Our overall findings thus suggest a ubiquitous nature of topography in spatial organization of functional connectivity among closely related functional regions in the human brain.

\section{Eccentricity-dependent functional connectivity in early visual cortex as a special case of linear communication}

We found that the early visual cortex demonstrates orderly region-to-region functional connectivity that maintains the organization of eccentricity representation across regions. This result replicates previous work that used resting state $\mathrm{fMRI}$ data and phase-locking fMRI task data in studies of retinotopic organization of the visual system (Haak et al., 2013; Gravel et al., 2014; Arcaro et al., 2015). The orderly functional connectivity likely contributes to the maintenance of retinotopic organization across the posterior cortical regions for multiple levels of visual processing. We also demonstrated that this motif breaks down for connectivity between early and higher order visual cortices (Fig. 4). The pattern of FFA connectivity demonstrates predicted qualities given its receptive field properties, that is, its convergence and selection of foveal resources (Kanwisher et al., 1997). These findings on region-to-region connectivity patterns provide a potential structure for understanding what information is maintained across regions, which would be useful for future modeling of information transformation and transfer from one region to another region (Thivierge and Marcus, 2007; Basti et al., 2018).

The topographic organization of functional connectivity observed in the early visual cortices seems to be a special case of a larger phenomenon. Our findings suggest that such a spatial layout of functional connectivity is a probable general mode of communication across the brain (Figs. 5, 6), likely at least partially for the purposes of highfidelity information transmission across regions within a functional network (Thivierge and Marcus, 2007). In the visual cortex, coincidental activity that drives plasticity provides a potential mechanism for the generation and maintenance a spatially linear organization of communication between nearby regions (Schoen et al., 1990; Elliott and Shadbolt, 1996; Catalano et al., 1997). However, activity dependent synaptic pruning is not the only mechanism that drives organization in early visual cortex. Recent studies have suggested a form of protoorganization driven by genetic expression and their products (e.g., morphogens) in animal models of development and in humans (Cheng et al., 1995; Crowley and Katz, 2000; Arcaro and Livingstone, 2017). This mechanism of organization developing from molecular gradients is more widely observed, pertaining to many brain systems. For example, molecular signaling has been shown to be responsible for organization of thalamocortical connections in and ordered spatial gradient, with Emx2 and Pax2 regulating the anterior-posterior organization of cortical fields and their thalamic afferents (Bishop et al., 2000). Using 3$D$ connective field modeling, we found a strong linear organization in lateral prefrontal areas despite a lack of clear or consistent retinotopy in these areas (Hagler and Sereno, 2006; Kastner et al., 2007). Strong linearity in the absence of retinotopy suggests a potential link with proto-organization. Indeed, our gradient decomposition of linearity resembles gradients of genetic expression (Burt et al., 2018; Murray et al., 2018) providing potential convergent evidence for this interpretation.

\section{Topographic connectivity and computation within and across large-scale brain networks}

Spatially linear organization of input-output relationships is thought to maintain information across levels of processing, allowing for precise action on continuous 
sensory information (Thivierge and Marcus, 2007). Topographic organization and projections from topographic space has been postulated to produce ordered abstract space as shown in connectionist models (Tinsley, 2009). With a continuous space of abstract relations, complex information transformation in abstract space can be achieved by the same general mechanism as filtering of visual information. For example, this is the proposed mechanism underlying saliency map models, where information from many independent channels are combined topographically to represent the overall visual salience of an image (Itti and Koch, 2000; Roggeman et al., 2010). These saliency map models have been successful in predicting eye movements to complex visual stimuli, attentional load responses in parietal cortex, and even working memory capacity (Foulsham and Underwood, 2008; Knops et al., 2014).

With the simple assumption information is spatially segregated, a tractable form of general computation in the brain is through spatial biases in connectivity, which would subsume the above description of topographic connectivity. Spatial biases can, however, have vastly different scopes. With distinct information converging onto a neuron giving rise to many potential combinations of that information, it has been shown that the exact combination is at least partially determined by spatial biases in convergence along the dendritic compartment (Taylor et al., 2000; London and Häusser, 2005). Higher order spatial biases, from cortical column to cortical column, may be responsible for higher order computation. Previous work has examined information transformation more directly, estimating linear transformations that map the representational dissimilarity matrices from one region to another (Basti et al., 2018). Our 3-D connective field model provides an appropriate description of the proposed mechanisms for these transformations, allowing for critical tests of this theory in observational fMRI data.

\section{Organization of the lateral prefrontal cortex using spatial connectivity metrics}

Gradient decomposition of topographic connectivity of the prefrontal functional connectivity demonstrated evidence for two gradients of functional organization. Gradient 2, particularly, seemed to predict task based activational differences between face and scene perception. Previous studies have consistently found differential projections from dorsal and ventral visual stream nodes to dorsal and ventral prefrontal cortex, respectively (Kawamura and Naito, 1984; Goldman-Rakic, 1987; Cavada and Goldman-Rakic, 1989). Indeed, scene processing, while involving specialized regions of the ventral visual pathway, does seem to also involve the dorsal stream (Aminoff and Tarr, 2015). This segregation of dorsal and ventral pathway input into the prefrontal cortex has been supported both structurally and functionally in humans (Takahashi et al., 2013). This dorsal-ventral segregation of the prefrontal cortex has been shown to be differentially responsible for spatial and object working memory (Constantinidis and Qi, 2018). Indeed, inactivation of dorsal prefrontal cortex impairs spatial working memory, but not object working memory
(Chafee and Goldman-Rakic, 2000; Suzuki and Gottlieb, 2013; Clark et al., 2014). Thus, the differential input from dorsal and ventral streams has functional consequences, leading to the segregation of downstream dependence of higher order functions depending on the type of information being operated on.

While a prominent perspective of prefrontal organization, the domain specialization hypothesis of lateral prefrontal functional organization is not the only studied organizational principle. Accumulating evidence shows another prominent gradient of prefrontal functional organization along the anterior-posterior axis related to the level of abstraction, from the posterior representation of sensorimotor response selection to the more abstract representation of task goals/rules in the anterior portions of the prefrontal cortex (Koechlin et al., 2003; Kouneiher et al., 2009; Azuar et al., 2014). By varying the rules and contexts of various tasks, it was found that areas related to the stimulus response mapping were located more caudally, while areas related to contexts and rules were more rostral (Koechlin et al., 2003; Badre and D'Esposito, 2007; Azuar et al., 2014). While we found that convergence follows a posterior to anterior gradient, there was no evidence that it related to the rule contrast in the mixed task. Patterns of convergence in the lateral prefrontal cortex were, however, associated with different sensory information, likely relating to differential processing demands required. Topographic gradient 2, on the other hand, did strongly related to the rule contrast in the mixed task, suggesting that topographic connectivity within the lateral prefrontal cortex delineates multiple pathways of organization.

In sum, on the basis of these new metrics of the spatial profile of functional connectivity, our data support both frameworks of functional organization for the lateral prefrontal cortex. However, based on these results it may be the case that these two functionally defined organizations are a result of the same underlying neural organization, driven by the differential topographic input within the lateral frontal cortex. This is consistent with the previous literature examining functional connectivity which has found evidence for both dorsal-ventral and rostral-caudal organizations in the lateral prefrontal cortex (Blumenfeld et al., 2013; Schumacher et al., 2019).

\section{Limitations and future directions}

Our estimations of topographic organization of connectivity between across regions were likely subjected to parcel selection, which is a limitation for this type of analysis. Given the multitude of parcellations and the multitude of methods used to obtain parcellations, the results could look slightly different if other justified parcellations were used. It is likely that our estimations of linearity would be even stronger with "true" parcels, given that more specification in early visual cortex actually boosted the estimated linearity (from $\sim 40 \%$ to $\sim 60 \%$ ). In addition, the 3D. Gaussian model itself also has limitations and makes certain assumptions. For one, it assumes a single focal point of connectivity from one region to another, which is probably overly simplified. However, the final $R^{2}$ of the 
optimization procedure provides a marker for the fit of the model to the data; if these values are too low in the analysis, it would be inappropriate to make any conclusions about the fitted values. As the $R^{2}$ of the model tended to increase with more scan time, a large proportion of the misfit is likely due to noise. With the 30 -min sessions of the MSC data, the modal $R^{2}$ tended around $0.30-0.60$, indicating a reasonable fit. Gaussian mixture models can be developed in the future to account some conditions of misfit, such as regions with multiple maxima.

It is important to note that the 3-D connective field method has a high dependence on high signal-to-noise ratio in single voxels, as opposed to the more conventional analyses that either use signal averaging within ROls or smoothing to increase the signal-to-noise ratio. Because of this, higher resolution datasets may suffer from less stability due to the increase of proportion of thermal noise in each voxel (Edelstein et al., 1986), although the impact on signal-to-noise ratio can potentially be counterbalanced by a reduction in physiological noise (Bodurka et al., 2007). Further studies are needed to test our approach on higher resolution data. Optimally, the goal is to achieve a reliable connective field mapping at the size of functional columns of the ROls, which may be feasible in the frontal cortex, where columns may be as large as $800-$ $900 \mu \mathrm{m}$ (Hirata and Sawaguchi, 2008; Masse et al., 2017).

\section{Conclusions}

By applying 3-D connective field modeling on restingstate fMRI data, we demonstrated that topographic organization of functional connectivity is likely common mode of communication across the cerebral cortex. This pattern of connectivity organization is most evident within the same functional network module, with the whole brain illustrating a primary gradient of linearity of functional connectivity comparable to two different data sources: resting-state functional connectivity and genetic expression. By the same principles, the topographic connectivity of the lateral prefrontal cortex seemed to be organized around two axes: posterior superior frontal gyrus $\rightarrow$ anterior inferior frontal gyrus and posterior middle frontal gyrus $\rightarrow$ anterior inferior frontal gyrus/frontopolar cortex. Both these axes segregated spatial patterns of activation relating to domain specialization (spatial $\rightarrow$ object) and abstraction $(S-R \rightarrow$ rule). These findings show that deriving finer scale (voxel level) spatial organization of region-toregion connectivity in volumetric space seems to be useful in resting-state fMRI data. Vector field representations of region-to-region connectivity has the potential to be an informative visualization for information transfer and transformation from one region to another, with topographic connectivity and convergence of connectivity as the quantitative metrics, especially if the voxel size can be reduced to the size of a cortical column in the ROls.

\section{References}

Aminoff EM, Tarr MJ (2015) Associative processing is inherent in scene perception. PLoS One 10:e0128840.
Arcaro MJ, Livingstone MS (2017) A hierarchical, retinotopic protoorganization of the primate visual system at birth. Elife 6:e26196.

Arcaro MJ, McMains SA, Singer BD, Kastner S (2009) Retinotopic organization of human ventral visual cortex. J Neurosci 29:1063810652.

Arcaro MJ, Honey CJ, Mruczek RE, Kastner S, Hasson U (2015) Widespread correlation patterns of fMRI signal across visual cortex reflect eccentricity organization. Elife 4:e03952.

Azuar C, Reyes P, Slachevsky A, Volle E, Kinkingnehun S, Kouneiher F, Bravo E, Dubois B, Koechlin E, Levy R (2014) Testing the model of caudo-rostral organization of cognitive control in the human with frontal lesions. Neuroimage 84:1053-1060.

Badre D, D'Esposito M (2007) Functional magnetic resonance imaging evidence for a hierarchical organization of the prefrontal cortex. J Cogn Neurosci 19:2082-2099.

Baldassano C, Iordan MC, Beck DM, Fei-Fei L (2012) Voxel-level functional connectivity using spatial regularization. Neuroimage 63:1099-1106.

Basti A, Mur M, Kriegeskorte N, Pizzella V, Marzetti L, Hauk O (2018) Analysing linear multivariate pattern transformations in neuroimaging data. BioRxiv 497180.

Behzadi Y, Restom K, Liau J, Liu TT (2007) A component based noise correction method (CompCor) for BOLD and perfusion based fMRI. Neuroimage 37:90-101.

Bishop KM, Goudreau G, O'Leary DD (2000) Regulation of area identity in the mammalian neocortex by Emx2 and Pax6. Science 288:344-349.

Biswal BB, Mennes M, Zuo XN, Gohel S, Kelly C, Smith SM, Beckmann CF, Adelstein JS, Buckner RL, Colcombe S, Dogonowski AM, Ernst M, Fair D, Hampson M, Hoptman MJ, Hyde JS, Kiviniemi VJ, Kötter R, Li SJ, et al. (2010) Toward discovery science of human brain function. Proc Natl Acad Sci USA 107:4734-4739.

Blumenfeld RS, Nomura EM, Gratton C, D'Esposito M (2013) Lateral prefrontal cortex is organized into parallel dorsal and ventral streams along the rostro-caudal axis. Cereb Cortex 23:24572466.

Bodurka J, Ye F, Petridou N, Murphy K, Bandettini PA (2007) Mapping the MRI voxel volume in which thermal noise matches physiological noise-implications for fMRI. Neuroimage 34:542549.

Burt JB, Demirtaş M, Eckner WJ, Navejar NM, Ji JL, Martin WJ, Bernacchia A, Anticevic A, Murray JD (2018) Hierarchy of transcriptomic specialization across human cortex captured by structural neuroimaging topography. Nat Neurosci 21:1251-1259.

Byrd RH, Gilbert JC, Nocedal J (2000) A trust region method based on interior point techniques for nonlinear programming. Math Program 89:149-185.

Catalano SM, Chang CK, Shatz CJ (1997) Activity-dependent regulation of NMDAR1 immunoreactivity in the developing visual cortex. J Neurosci 17:8376-8390.

Cavada C, Goldman-Rakic PS (1989) Posterior parietal cortex in rhesus monkey: I. Evidence for segregated corticocortical networks linking sensory and limbic areas with the frontal lobe. J Comp Neurol 287:422-445.

Cavada C, Goldman-Rakic PS (1991) Topographic segregation of corticostriatal projections from posterior parietal subdivisions in the macaque monkey. Neuroscience 42:683-696.

Chafee MV, Goldman-Rakic PS (2000) Inactivation of parietal and prefrontal cortex reveals interdependence of neural activity during memory-guided saccades. J Neurophysiol 83:1550-1566.

Cheng HJ, Nakamoto M, Bergemann AD, Flanagan JG (1995) Complementary gradients in expression and binding of ELF-1 and Mek4 in development of the topographic retinotectal projection map. Cell 82:371-381.

Clark KL, Noudoost B, Moore T (2014) Persistent spatial information in the FEF during object-based short-term memory does not contribute to task performance. J Cogn Neurosci 26:1292-1299. 
Constantinidis C, Qi XL (2018) Representation of spatial and feature information in the monkey dorsal and ventral prefrontal cortex. Front Integr Neurosci 12:31.

Crowley JC, Katz LC (2000) Early development of ocular dominance columns. Science 290:1321-1324.

Di X, Gohel S, Kim EH, Biswal BB (2013) Task vs. rest-different network configurations between the coactivation and the restingstate brain networks. Front Hum Neurosci 7:493.

Dumoulin SO, Wandell BA (2008) Population receptive field estimates in human visual cortex. Neuroimage 39:647-660.

Edelstein WA, Glover GH, Hardy CJ, Redington RW (1986) The intrinsic signal-to-noise ratio in NMR imaging. Magn Reson Med 3:604618.

Elliott T, Shadbolt NR (1996) A mathematical model of activity-dependent, anatomical segregation induced by competition for neurotrophic support. Biol Cybern 75:463-470.

Fasano G, Franceschini A (1987) A multidimensional version of the Kolmogorov-Smirnov test. Mon Not R Astron Soc 225:155-170.

Foulsham T, Underwood G (2008) What can saliency models predict about eye movements? Spatial and sequential aspects of fixations during encoding and recognition. $J$ Vis 8:6-6.

Fox MD, Snyder AZ, Vincent JL, Corbetta M, Essen DCV, Raichle ME (2005) The human brain is intrinsically organized into dynamic, anticorrelated functional networks. Proc Natl Acad Sci USA 102:9673-9678.

Goldman-Rakic P (1987) Circuitry of the primate prefrontal cortex and the regulation of behavior by representational memory. In: Handbook of physiology, the nervous system, higher functions of the brain (Plum F, ed) Vol V, pp 373-417. Bethesda: American Physiological Society.

Goldman-Rakic PS (1999) The physiological approach: Functional architecture of working memory and disordered cognition in schizophrenia. Biol Psychiatry 46:650-661.

Gordon EM, Laumann TO, Gilmore AW, Newbold DJ, Greene DJ, Berg JJ, Ortega M, Hoyt-Drazen C, Gratton C, Sun H, Hampton JM, Coalson RS, Nguyen AL, McDermott KB, Shimony JS, Snyder AZ, Schlaggar BL, Petersen SE, Nelson SM, Dosenbach NUF (2017) Precision functional mapping of individual human brains. Neuron 95:791-807.e7.

Gravel N, Harvey B, Nordhjem B, Haak KV, Dumoulin SO, Renken R, Curcõi冈-Blake B, Cornelissen FW (2014) Cortical connective field estimates from resting state fMRI activity. Front Neurosci 8:339.

Haak KV, Winawer J, Harvey BM, Renken R, Dumoulin SO, Wandell BA, Cornelissen FW (2013) Connective field modeling. Neuroimage 66:376-384.

Haber SN, Fudge JL, McFarland NR (2000) Striatonigrostriatal pathways in primates form an ascending spiral from the shell to the dorsolateral striatum. J Neurosci 20:2369-2382.

Hagler DJ, Sereno MI (2006) Spatial maps in frontal and prefrontal cortex. Neuroimage 29:567-577.

Hallquist MN, Hwang K, Luna B (2013) The nuisance of nuisance regression: Spectral misspecification in a common approach to resting-state fMRI preprocessing reintroduces noise and obscures functional connectivity. Neuroimage 82:208-225.

Hirata Y, Sawaguchi T (2008) Functional columns in the primate prefrontal cortex revealed by optical imaging in vitro. Neurosci Res 61:1-10.

Hoover JE, Strick PL (1993) Multiple output channels in the basal ganglia. Science 259:819-821.

Hubel DH, Wiesel TN (1962) Receptive fields, binocular interaction and functional architecture in the cat's visual cortex. J Physiol 160:106-154.

Itti L, Koch C (2000) A saliency-based search mechanism for overt and covert shifts of visual attention. Vision Res 40:1489-1506.

Kanwisher N, McDermott J, Chun MM (1997) The fusiform face area: A module in human extrastriate cortex specialized for face perception. J Neurosci 17:4302-4311.

Kastner S, DeSimone K, Konen CS, Szczepanski SM, Weiner KS, Schneider KA (2007) Topographic maps in human frontal cortex revealed in memory-guided saccade and spatial working-memory tasks. J Neurophysiol 97:3494-3507.

Kawamura K, Naito J (1984) Corticocortical projections to the prefrontal cortex in the rhesus monkey investigated with horseradish peroxidase techniques. Neurosci Res 1:89-103.

Kay KN, Yeatman JD (2017) Bottom-up and top-down computations in word-and face-selective cortex. Elife 6:e22341.

Kendall DG (1989) A survey of the statistical theory of shape. Statist Sci 4:87-99.

Knops A, Piazza M, Sengupta R, Eger E, Melcher D (2014) A shared, flexible neural map architecture reflects capacity limits in both visual short-term memory and enumeration. J Neurosci 34:98579866.

Koechlin E, Ody C, Kouneiher F (2003) The architecture of cognitive control in the human prefrontal cortex. Science 302:1181-1185.

Kouneiher F, Charron S, Koechlin E (2009) Motivation and cognitive control in the human prefrontal cortex. Nat Neurosci 12:939-945.

Kravitz DJ, Saleem KS, Baker CI, Mishkin M (2011) A new neural framework for visuospatial processing. Nat Rev Neurosci 12:217230.

Kravitz DJ, Saleem KS, Baker CI, Ungerleider LG, Mishkin M (2013) The ventral visual pathway: An expanded neural framework for the processing of object quality. Trends Cogn Sci 17:26-49.

London M, Häusser M (2005) Dendritic computation. Annu Rev Neurosci 28:503-532.

Margulies DS, Ghosh SS, Goulas A, Falkiewicz M, Huntenburg JM, Langs G, Bezgin G, Eickhoff SB, Castellanos FX, Petrides M, Jefferies E, Smallwood J (2016) Situating the default-mode network along a principal gradient of macroscale cortical organization. Proc Natl Acad Sci USA 113:12574-12579.

Masse NY, Hodnefield JM, Freedman DJ (2017) Mnemonic encoding and cortical organization in parietal and prefrontal cortices. $J$ Neurosci 37:6098-6112.

Murray JD, Demirtaș M, Anticevic A (2018) Biophysical modeling of large-scale brain dynamics and applications for computational psychiatry. Biol Psychiatry Cogn Neurosci Neuroimaging 3:777787.

Petrides M (2005) Lateral prefrontal cortex: Architectonic and functional organization. Philos Trans R Soc Lond B Biol Sci 360:781795.

Roggeman C, Fias W, Verguts T (2010) Salience maps in parietal cortex: Imaging and computational modeling. Neuroimage 52:10051014.

Saygin AP, Sereno MI (2008) Retinotopy and attention in human occipital, temporal, parietal, and frontal cortex. Cereb Cortex 18:2158-2168.

Schaefer A, Kong R, Gordon EM, Laumann TO, Zuo X-N, Holmes AJ, Eickhoff SB, Yeo BTT (2018) Local-global parcellation of the human cerebral cortex from intrinsic functional connectivity MRI. Cereb Cortex 28:3095-3114.

Schoen SW, Leutenecker B, Kreutzberg GW, Singer W (1990) Ocular dominance plasticity and developmental changes of 5'-nucleotidase distributions in the kitten visual cortex. J Comp Neurol 296:379-392.

Schumacher FK, Schumacher LV, Schelter BO, Kaller CP (2019) Functionally dissociating ventro-dorsal components within the rostro-caudal hierarchical organization of the human prefrontal cortex. Neuroimage 185:398-407.

Smith SM, Jenkinson M, Woolrich MW, Beckmann CF, Behrens TEJ, Johansen-Berg H, Bannister PR, De Luca M, Drobnjak I, Flitney DE, Niazy RK, Saunders J, Vickers J, Zhang Y, De Stefano N, Brady JM, Matthews PM (2004) Advances in functional and structural MR image analysis and implementation as FSL. Neuroimage 23:S208-S219.

Suzuki M, Gottlieb J (2013) Distinct neural mechanisms of distractor suppression in the frontal and parietal lobe. Nat Neurosci 16:98104.

Takahashi E, Ohki K, Kim D-S (2013) Dissociation and convergence of the dorsal and ventral visual working memory streams in the human prefrontal cortex. Neuroimage 65:488-498. 
Tanaka K (1997) Mechanisms of visual object recognition: Monkey and human studies. Curr Opin Neurobiol 7:523-529.

Taylor WR, He S, Levick WR, Vaney DI (2000) Dendritic computation of direction selectivity by retinal ganglion cells. Science 289:23472350.

Thivierge J-P, Marcus GF (2007) The topographic brain: From neural connectivity to cognition. Trends Neurosci 30:251-259.

Tinsley CJ (2009) Creating abstract topographic representations: Implications for coding, learning and reasoning. Biosystems 96:251258.

Ungerleider LG, Courtney SM, Haxby JV (1998) A neural system for human visual working memory. Proc Natl Acad Sci USA 95:883890.

Van Essen DC, Gallant JL (1994) Neural mechanisms of form and motion processing in the primate visual system. Neuron 13:110.

Wang L, Mruczek REB, Arcaro MJ, Kastner S (2015) Probabilistic maps of visual topography in human cortex. Cereb Cortex 25:3911-3931.
Whitfield-Gabrieli S, Nieto-Castanon A (2012) Conn: A functional connectivity toolbox for correlated and anticorrelated brain networks. Brain Connect 2:125-141.

Wolpert DM, Flanagan JR (2001) Motor prediction. Curr Biol 11: R729-R732.

Yarkoni T, Poldrack RA, Nichols TE, Van Essen DC, Wager TD (2011) Large-scale automated synthesis of human functional neuroimaging data. Nat Methods 8:665-670.

Yeo BTT, Krienen FM, Sepulcre J, Sabuncu MR, Lashkari D, Hollinshead M, Roffman JL, Smoller JW, Zöllei L, Polimeni JR, Fischl B, Liu H, Buckner RL (2011) The organization of the human cerebral cortex estimated by intrinsic functional connectivity. J Neurophysiol 106:1125-1165.

Zuo XN, Anderson JS, Bellec P, Birn RM, Biswal BB, Blautzik J, Breitner JCS, Buckner RL, Calhoun VD, Castellanos FX, Chen A, Chen B, Chen J, Chen X, Colcombe SJ, Courtney W, Craddock RC, Di Martino A, Dong HM, Fu X, et al. (2014) An open science resource for establishing reliability and reproducibility in functional connectomics. Sci Data 1:140049. 